\title{
Segurança dos alimentos em serviços de alimentação do setor de turismo
}

\author{
Marcel Levy de Andrade ${ }^{1}$ e Gilma Lucazechi Sturion ${ }^{2}$
}

O setor de turismo no Brasil está em expansão devido, principalmente, à inclusão do país na agenda internacional de grandes eventos. Esses eventos são caracterizados pelo aumento da demanda dos turistas e pela procura de serviços relacionados ao setor. $\mathrm{O}$ deslocamento dos indivíduos e a maior exposição da população aos alimentos destinados ao pronto consumo em serviços de alimentação contribuem para a incidência de Doenças Transmitidas por Alimentos (DTA). Surtos de origem alimentar, geralmente, estão associados ao conhecimento insatisfatório dos manipuladores em relação às práticas adequadas de higiene em diferentes ramos de serviços de alimentação, inclusive no setor de turismo. Além disso, outros fatores como recrutamento inadequado de pessoal, insuficiente capacitação técnica de gerentes e manipuladores e pouco interesse e comprometimento dos gestores em desenvolver programas da qualidade e segurança dos alimentos, aumentam os riscos à saúde dos consumidores. Por isso, é importante verificar quais as necessidades e dificuldades dessas empresas para caracterizar e direcionar os esforços e diminuir as não conformidades encontradas neste setor. Nesse contexto, o objetivo do trabalho é caracterizar os serviços de alimentação do setor de turismo em relação aos problemas enfrentados pelas empresas no tocante à segurança dos alimentos.

Palavras-chave: serviços de alimentação, turismo, segurança dos alimentos, manipuladores de alimentos, recursos humanos.

\section{Food safety in food services of the tourism sector}

The tourism sector in Brazil is increasing due mainly to the inclusion of the country in the international calendar of the major events. These events are characterized by an increase in demand by tourists and services related to the sector. The displacement of individuals and the greater exposure of the population to ready-to-eat foods services, contribute to the incidence of Foodborne Diseases (FBD). Usually, foodborne disease outbreaks are associated with unsatisfactory knowledge of the handlers in relation to appropriate hygiene practices in different food services, including the tourism sector. In addition, other factors such as inappropriate recruiting, insufficient technical training of managers and food handlers and little interest and commitment of managers in developing quality programs and food safety, increase the risks to consumer health. Therefore, it is important to verify the needs and difficulties of these companies to characterize and direct efforts and reduce nonconformities found in this sector. In this context, the aim of this study was to characterize the tourism food services in relation to the problems related to food safety in the companies.

Key-words: food services, tourism, food safety, food handlers, resource human.

\footnotetext{
${ }^{1}$ Departamento de Agroindústria, Alimentos e Nutrição, Universidade de São Paulo (USP), Escola Superior de Agricultura "Luiz de Queiroz" (ESALQ). Correspondência: Av. Pádua Dias nô11, CEP 13418-900, Piracicaba, São Paulo, Brasil. Telefone: (19)3429-4150. E-mail:ml_andrade@yahoo.com.br

2 Profa. Dra. Departamento de Agroindústria, Alimentos e Nutrição, Universidade de São Paulo (USP), Escola Superior de Agricultura "Luiz de Queiroz" (ESALQ). Correspondência: Av. Pádua Dias no 11, CEP 13418-900, Piracicaba, São Paulo, Brasil. E-mail: glsturio@usp.br 


\section{INTRODUÇÃO}

O número de refeições fora do domicílio encontra-se em expansão no Brasil [1] com um crescimento, no período entre 2002 e 2010, de 24\% para $31 \%$ [2,3]. A tendência observada no país ainda está aquém dos índices europeus e norte-americanos, onde este setor é responsável por 50\% a 60\% do consumo de alimentos [4].

Atualmente, o consumo fora do domicílio no Brasil alcança índices de $34 \%$ e o setor conta com unidades de produção de portes e tipos de organização diferentes, como restaurantes comerciais, industriais e institucionais, bufês, hotéis, fast-foods, catering e outros [4, 5].

De 2003 a 2012, a participação do turismo no Produto Interno Bruto (PIB) nacional triplicou e o faturamento do setor em 2013, cresceu 4,8\%, o dobro do PIB do setor de serviços [ø. Este crescimento pode ser justificado pela inserção do país na agenda internacional de grandes eventos, como World Cup FIFA 2014 e Olimpic Games de 2016 [7].

A maioria das empresas de serviços de alimentação no país é de micro e pequeno porte, inclusive aquelas envolvidas com o turismo [8]. Estas empresas apresentam dificuldades em desenvolver programas direcionados à segurança de alimentos, devido à deficiência de recursos financeiros, humanos e de infraestrutura [9]. Estas condições potencializam os problemas relacionados às práticas de higiene inadequadas.

Surtos de origem alimentar, geralmente, estão associados ao conhecimento insatisfatório dos manipuladores sobre as práticas adequadas de higiene em diferentes ramos de serviços de alimentação como restaurantes [10], empresas de catering [11], instituições [12] hotéis [13] e dos consumidores [14]. Esses fatos demonstram a necessidade de formação continua em higiene na manipulação dos agentes envolvidos no setor e, aos órgãos públicos e autoridades sanitárias, em realizar ações educativas e de conscientização como forma de apoio as empresas e aos consumidores visando o preparo e consumo de alimentos seguros ${ }^{[15]}$. Somamse a esses fatores a falta de inspeções regulares dos órgãos fiscalizadores e a capacitação inadequada dos agentes sanitários $[16,17]$.
Dentre os fatores que também contribuem para o maior número de ocorrência de DTA destacam-se, o aumento populacional, a existência de grupos populacionais vulneráveis, o processo de urbanização desordenado, a necessidade de produção de alimentos em grande escala e as facilidades de deslocamento dos indivíduos $[1,5]$.

No Brasil, a produção de alimentos livres de contaminação tem sido amplamente discutida devido ao aumento do consumo ${ }^{[1,18]}$ que vem acompanhado por alterações de hábitos alimentares (comer fora do domicilio, entre outras) aliadas às mudanças impostas pela globalização. O desenvolvimento do hábito de comer fora do domicilio pode ser analisado a partir das seguintes perspectivas: a alimentação relacionada com uma atividade social (lazer e interação social), o distanciamento entre o local de trabalho e a residência, $o$ crescimento de alimentos industrializados e a necessidade imposta pelo modelo de força de trabalho, em que a mulher passou a ter papel relevante ${ }^{[1,5]}$.

A inserção das mulheres no mercado de trabalho contribuiu para a construção de uma sociedade mais igualitária, tanto nos espaços reprodutivos (família) como nos espaços produtivos (trabalho) [19]. As mulheres passaram a desempenhar um papel muito mais relevante do que os homens no crescimento da população economicamente ativa (PEA), aumentando de 44,4\% para 46,1\%, entre 2003 e 2011 [20]. O distanciamento da mulher das atividades tradicionais desenvolvidas no lar, associado ao aumento na taxa de urbanização das populações, com incremento na renda familiar, contribuíram para o processo de transição nutricional pelo qual vêm passando as sociedades desenvolvidas [21].

O desenvolvimento de uma revisão bibliográfica relacionada à caracterização de serviços de alimentação do setor de turismo poderá fornecer subsídios ao desenvolvimento de estratégias institucionais de intervenção direcionadas à segurança dos alimentos. Além disso, proporcionar uma melhor compreensão do comportamento dos manipuladores nas operações que representam riscos à saúde dos consumidores, bem como, contribuir para a realização de pesquisas futuras na área visando suprir a lacuna na literatura sobre o assunto neste setor. 


\section{OBJETIVO}

Esta revisão visa caracterizar os serviços de alimentação do setor de turismo quanto a sua representatividade no segmento e aspectos relacionados à segurança dos alimentos.

\section{METODOLOGIA}

O presente trabalho constituiu-se como uma revisão bibliográfica utilizando como plataforma de pesquisa Scielo, Portal Capes, Portais governamentais e de instituições públicas nacionais e internacionais, Google Acadêmico, Scopus, Science Direct e bases de pesquisa da USP (Sistema de Busca integrado de Bibliotecas - SIBI e Biblioteca Digital - Teses). Para a realização buscou-se documentos do tipo artigo científico, capítulos de livros, documentos de âmbito nacional e internacional, e documentos em formato eletrônico, compreendendo o período de 2000 a 2014. O levantamento bibliográfico sobre o assunto foi descrito sob cinco temas: Serviços de Alimentação, Turismo, Segurança dos Alimentos, Manipuladores de Alimentos e Recursos Humanos.

\section{Canais de distribuição de serviços de alimentação}

Dados do IBGE [22] mostram que o setor de serviços de alimentação no Brasil contava com 193.309 unidades em operação, distribuídos por todo o território nacional, oferecendo ampla gama de produtos e serviços. No período entre 2007-2010 os serviços de alimentação (restaurantes e similares) foram os que tiveram maior crescimento do faturamento no acumulado, cerca de 50,4\%, em média, e a receita do grupo subiu $44,9 \%$ no período. Esses valores correspondem a uma receita operacional líquida de $\mathrm{R} \$ 55,7$ bilhões (64,5\%), com 1,4 milhões de pessoas ocupadas $(61,8 \%)$ representando $\mathrm{R} \$ 13,1$ bilhões $(60,2 \%)$ de pagamentos, entre salários, retiradas e outras remunerações, considerando todos os ramos de serviços no país [23].
As mais de 190 mil unidades de serviços de alimentação são representadas, em sua maioria, por Micro e Pequenas empresas (MPE) [22]. Esse tipo de empresa é considerado importante pela capacidade de gerar empregos ou contribuir para a produção industrial e de serviços. As empresas classificadas nas referidas categorias exercem um papel relevante na economia de seus países, pois não somente contribuem com a geração de riqueza, emprego e mão de obra, mas também afetam a capacidade das grandes empresas em serem competitivas [24, 25].

Do total de MPE no Brasil, 49,4\% estavam no segmento de comércio, $34,6 \%$ no segmento de serviços e 10,8\% no segmento industrial [8]. No Estado de São Paulo cerca de $40 \%$ da MPE atuam no segmento de serviços e destes 19,4\% representado pelos serviços de alimentação [26].

A Figura 1 mostra os diferentes canais envolvidos na distribuição de alimentos prontos para consumo, classificados de acordo com o padrão adotado pela ABIA [1], e o crescimento do faturamento das vendas da indústria de alimentos para os mesmos no período de 2004 a 2007. 
Figura 1. Faturamento (R\$ bilhões), no Brasil, das vendas da indústria de alimentos para os canais de distribuição de serviços de alimentação no período de 2004 e 2007.

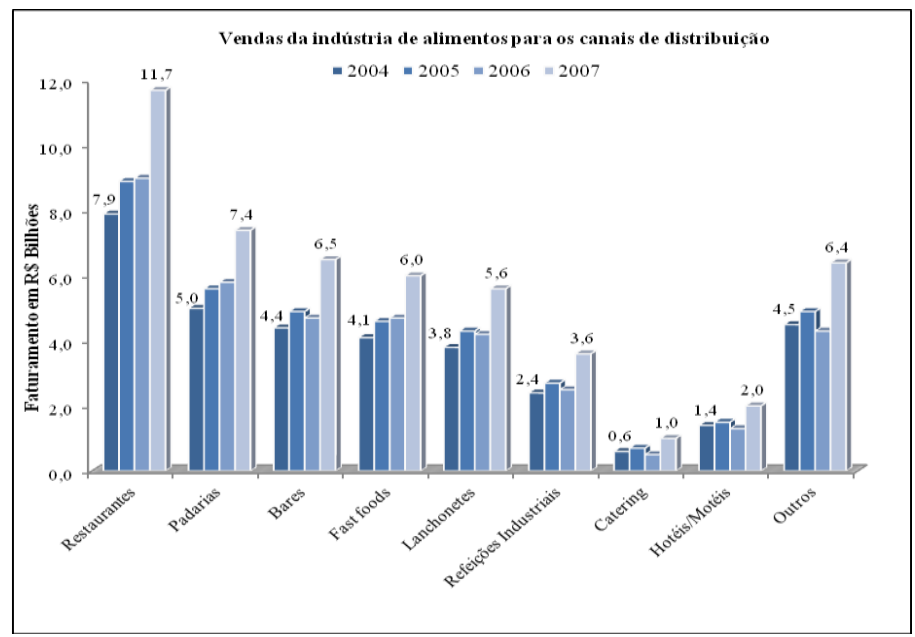

Fonte: ABIA 2006; 2008a; 2008b [27-29]

A Figura 2 apresenta o percentual de vendas, no Brasil, da indústria de alimentos para os canais de distribuição. Em 2008, os maiores percentuais de vendas foram direcionados para restaurantes comerciais $(23,0 \%)$, padarias $(15,0 \%)$, bares $(13 \%)$ e fast-foods $(12 \%)$. Observa-se que o faturamento das vendas, no período, cresceu significativamente em todos os setores ${ }^{[27-29]}$.

Figura 2. Percentual das vendas, no Brasil, da indústria de alimentos para os canais de distribuição no ano de 2008.

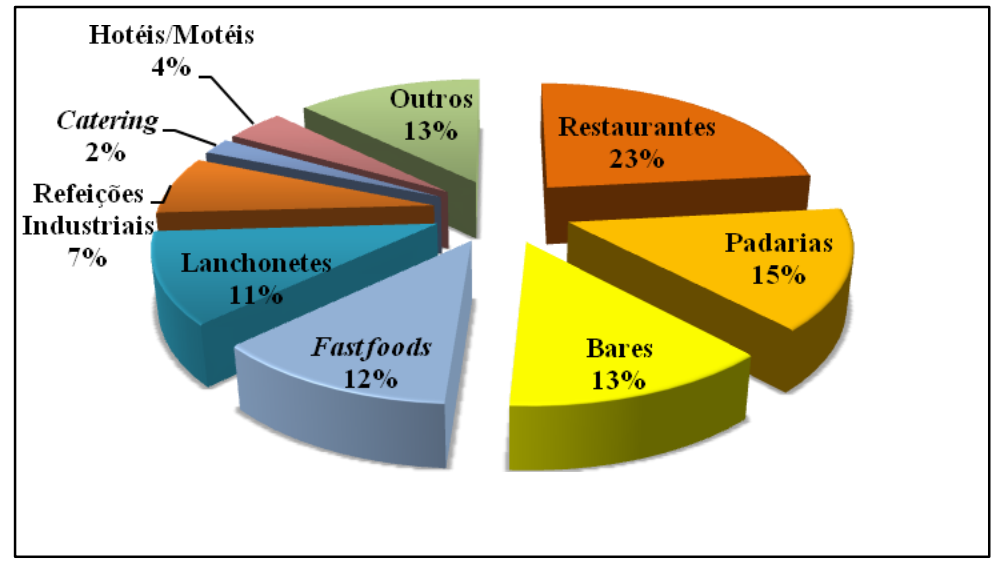

Fonte: ABIA 2008 [28, 29] 
A ABIA [30] registra que a venda de produtos da indústria de alimentos para canais de distribuição de serviços de alimentação passou de $\mathrm{R} \$ 38,6$ bilhões, em 2005, para R\$ 116,5 bilhões em 2013. Esses dados sugerem crescimento da demanda por consumo de alimentos prontos e o grande potencial econômico desse serviço.

Na Europa e nos EUA, 50\% a 60\% da venda de alimentos destinam-se aos canais de Serviços de alimentação, enquanto no Brasil este número ainda é de
$34 \%$, que revela grande potencial de expansão do setor [4].

A ABIA [1] destaca que, nos últimos dez anos as vendas para o setor de serviços de alimentação cresceram $14,7 \%$ ao ano, ante 10,8\% do setor de varejo, demonstrando sua representatividade. $\mathrm{O}$ crescimento desse setor foi expressivo nos últimos 5 anos e superior ao canal de varejo, conforme mostra a Figura 3.

Figura 3. Percentual de vendas da indústria de alimentos para o setor de varejo e serviços de alimentação no Brasil entre 2002-2012.

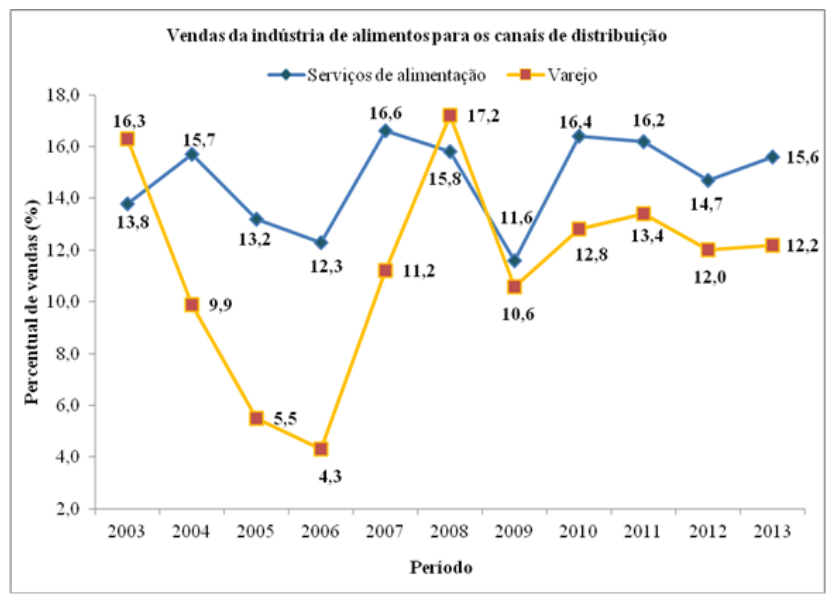

Fonte: ABIA $2014{ }^{[1]}$

É possível observar que as vendas para os canais de serviços de alimentação se mantiveram mais estáveis quando comparadas com as vendas para o varejo. Segundo a ABIA [1] a venda da indústria de alimentos para os diversos segmentos de serviços de alimentação chegou a R \$ 116,5 bilhões em 2013, que transformados pelos operadores e somados aos produtos in natura alcançaram $\mathrm{R} \$ 284,8$ bilhões.

\section{Serviços de alimentação do setor de turismo}

O setor de turismo é um ramo importante e com potencial de desenvolvimento dentro dos canais de distribuição de serviços de alimentação fora do domicílio, pois o hóspede alimenta-se necessariamente fora de casa ${ }^{[31]}$. De acordo com a Organização Mundial do Turismo, as atividades relacionadas geram emprego para 230 milhões de pessoas em todo o mundo, o que representa $8,7 \%$ dos postos de trabalho existentes [32].

A cadeia produtiva do turismo está em expansão, devido à inclusão do Brasil na agenda internacional de grandes eventos (Copa do Mundo de 2014 e Olimpíadas de 2016) [7,33]. Em 2012, a oferta de meios de hospedagem correspondia a 6,2 mil estabelecimentos com 331.833 mil unidades habitacionais e 686.495 leitos, distribuídos por todo o território nacional [34]. De 2003 a 2012 a participação do turismo no Produto Interno Bruto (PIB) nacional tripilou e o faturamento do setor em 2013 cresceu 4,8\%, o dobro dos $2,4 \%$ do PIB do setor de serviços ${ }^{[6]}$.

A Figura 4 demonstra os percentuais de meios de hospedagem no Brasil, de acordo com as regiões. 
Figura 4. Percentual de meios de hospedagem por região no ano de 2012, no Brasil.

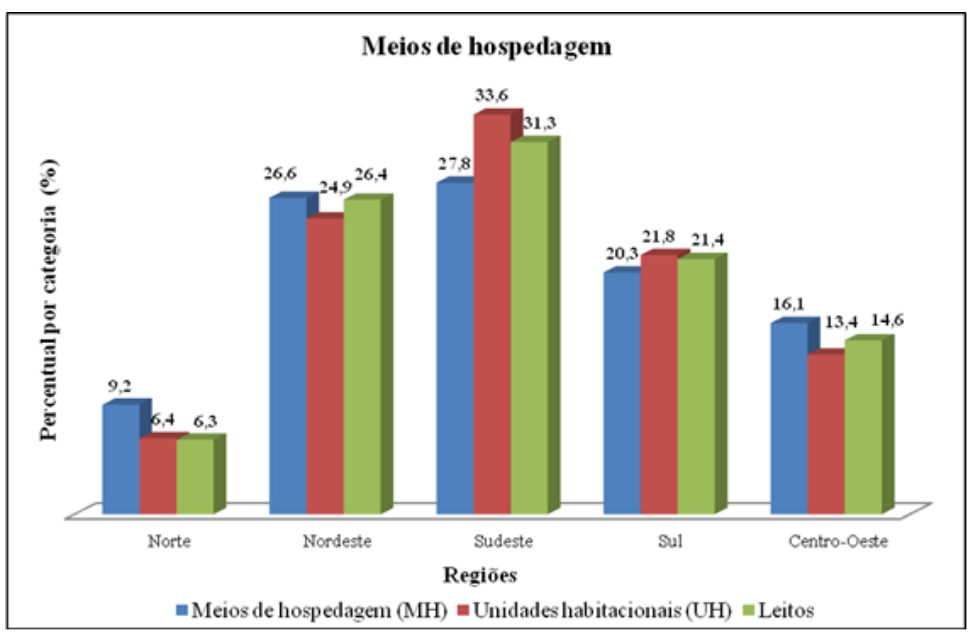

Fonte: Brasil 2014 [34]

Em 2012, os maiores percentuais de meios de hospedagem $(33,6 \%, \quad 23,6 \%$ e $20,3 \%)$, foram registrados respectivamente, nas regiões Sudeste, Nordeste e Sul do Brasil [34].

Atualmente, o Brasil está passando por um momento favorável para a expansão hoteleira e terá pelo menos 422 novos empreendimentos de hospedagem até 2016. O investimento privado deve ultrapassar a $\mathrm{R} \$ 12,2$ bilhões, gerando 33,8 mil novos empregos diretos no setor. A região Sudeste receberá a maior parte dos investimentos previstos (59\%), com 250 novos estabelecimentos, seguida pelo Nordeste $(13 \%)$, Sul (11\%), Centro-Oeste (10\%) e Norte (7\%) [7.

Em relação aos gastos com o turismo, no período entre 2008 e 2009, o transporte e a alimentação foram os itens de maior representatividade nas despesas com turismo das familias brasileiras. A participação média das duas variáveis no conjunto de gastos foi de $48,7 \%$ e $22,6 \%$, respectivamente [2]. O Ministério do Turismo [22] classifica as Atividades Características do Turismo (ACT) em: hotéis e outros serviços de alojamento, serviços de residência secundária por conta própria ou gratuita, serviços de alimentação, serviços de transporte ferroviário, rodoviário, aquaviário, aéreo, serviços de apoio, agências de viagens, operadores e guias turísticos, aluguel de veículos e serviços culturais, de recreação e lazer. Os estabelecimentos relacionados às ACT para serviços de alimentação são restaurantes, estabelecimentos de bebidas, com serviço completo, lanchonete e similares e demais tipos, dentre os quais, o de comida preparada para o público em geral em locais abertos, permanentes ou não, tais como: trailers, quiosques, carrocinhas e outros tipos de serviços ambulantes de alimentação preparada para consumo imediato [22].

Dentre os grupos do setor de serviços no Brasil, chama atenção a representatividade do setor de serviços de alimentação relacionado ao setor de turismo. A atividade de serviços de alimentação apresentou a maior participação na produção das ACT $(41,9 \%)$, seguido do transporte rodoviário, com $(16,3 \%)$ e das atividades recreativas e culturais com $13,6 \%$ do total do grupo. Os serviços de alimentação também registraram o maior número de ocupações: 3,0 milhões, ou 50,7\% do total, a maior participação nas remunerações sendo 29,0\% do grupo das ACT, porém, apresentou a menor remuneração média paga aos funcionários quando comparada as outras ACT. Os resultados demonstram que os serviços de alimentação no setor de turismo possuem alta representatividade, contudo, os salários pagos aos funcionários são baixos quando comparados à remuneração de outras atividades desse segmento [22]. 


\section{Segurança dos alimentos no setor de turismo}

Os restaurantes do setor de turismo, especialmente, das MPE, são muitas vezes desprovidos de profissionais com formação técnica ou superior específica na área de alimentos [13, 35]. Além disso, há restrições financeiras que limitam $\mathrm{o}$ acesso às informações, à capacitação dos funcionários e à adequação de infraestrutura, gerando falhas de conhecimento, entendimento e aplicação das exigências legais ou normativas pré-estabelecidas. Por isso, considerando essa perspectiva, o serviço de alimentação deve ser motivo de preocupação dentro da cadeia produtiva do turismo, uma vez que envolve desde os aspectos econômicos até os de segurança dos alimentos, imprescindíveis para a sua sustentabilidade e competitividade. Justifica-se, então, a necessidade de identificar e dimensionar as dificuldades e desafios do setor e, assim, direcionar ações que previnam com maior eficácia a possibilidade de ocorrência de Doenças Transmitidas por Alimentos (DTA) [13,36.

O mercado hoteleiro vem se expandindo a cada ano, porém a capacidade técnica dos manipuladores, referindo-se à higiene e à sanidade no preparo das refeições, não vem acompanhando essa expansão. Casos de DTA relacionados a eventos de massa já foram notificados na Copa do Mundo realizada na Alemanha em 2006 [37] e nos jogos Olímpicos de 2012 em Londres [38] relacionados a problemas de qualidade higiene-sanitária de restaurantes e aos alimentos vendidos por ambulantes que se instalam nos arredores dos eventos. Autores relatam que os potenciais perigos na preparação e venda de alimentos por ambulantes são, principalmente, devido à utilização de matérias-primas de baixa qualidade e as inspeções ineficazes [17]. Por isso é de suma importância que os consumidores permaneçam atentos às questões de higiene em relação aos serviços de alimentação, e que cobrem mais atitudes dos órgãos fiscalizadores na execução de ações que permitam garantir seu bem estar e a qualidade dos produtos e serviços consumidos. As ações de inspeção são consideradas pelos consumidores como atividades importantes, e acreditam que estes procedimentos deveriam ser realizados com maior frequência pelos órgãos fiscalizadores [39]. Uma grande preocupação dos responsáveis por uma Unidade Produtora de Refeições (UPR) é a produção de refeições seguras do ponto de vista higiênico-sanitário. De modo geral, as UPR recebem diariamente grandes quantidades de alimentos de alta perecibilidade, o que demanda tempo de estocagem, de manipulação e de exposição e muitas vezes sob temperaturas inadequadas [40].

Esses fatos favorecem a contaminação e multiplicação bacteriana no alimento, que ao ser consumido, pode desencadear alguma doença de origem alimentar. Sendo assim, é de suma importância que estes estabelecimentos utilizem ferramentas da qualidade, como Boas Práticas de Higiene e o sistema de Análise e Princípios e Pontos Críticos de Controle, como forma de gerenciar os riscos relacionados à contaminação dos alimentos. Além disso, pode ser um diferencial competitivo das empresas no mercado, pois além de oferecer serviços de qualidade (hospedagem, lazer e outros), também poderão proporcionar uma comida gastronomicamente desejável e segura, do ponto de vista higiênico-sanitário [41-44].

Estudos sobre avaliação das Boas Práticas em serviços de alimentação organizados pelo FDA [45] identificaram quais foram os maiores problemas (não conformidades) relacionados às praticas de higiene, como mostrado na Figura 5. 
Figura 5. Distribuição percentual de não conformidades encontradas em serviços de alimentação de refeições principais.

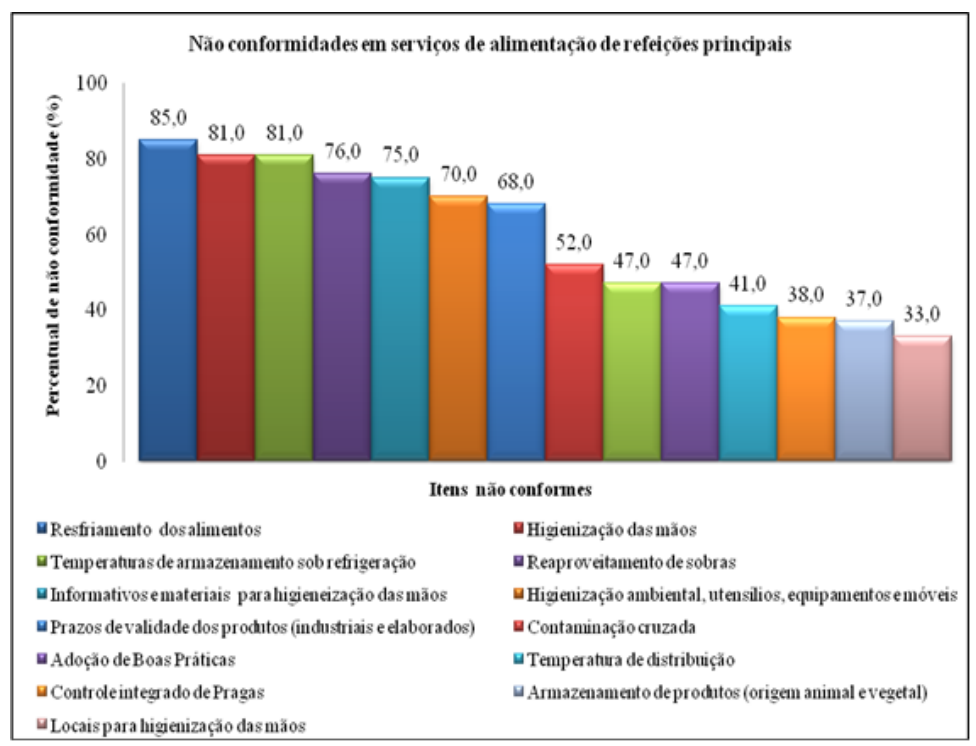

Fonte: FDA 2000 [45]

O objetivo do trabalho foi estabelecer uma linha base das empresas deste setor e regulamentar os esforços para mudar comportamentos e práticas diretamente relacionadas com Doenças Transmitidas por Alimentos (DTA). Verificou-se que os maiores problemas foram os relacionados à falta de higienização adequada das mãos (81\%) de cartazes informativos e materiais para execução (75\%), e aos aspectos de produção, ao resfriamento inadequado dos alimentos $(81 \%)$, ao reaproveitamento de sobras alimentares $(76 \%)$ e ao controle do prazo de validade dos produtos $(68 \%)$.

Algumas não conformidades verificadas pelo FDA [45] também foram evidenciadas por outros autores que constataram inadequações na técnica e frequência de higienização das mãos por manipuladores, no armazenamento, na uniformização, nas instalações, nas temperaturas de distribuição e acondicionamento (espera), contaminação cruzada, e ausência do controle integrado de pragas. ${ }^{[46-52]}$.

Estudos mais recentes indicam que os problemas citados ainda são recorrentes em serviços de alimentação devido ao conhecimento insuficiente dos manipuladores em relação às práticas de higiene adequadas e, por isso, enfatizam a necessidade das empresas em investir em treinamentos periódicos e contínuos como forma de amenizar os problemas em segurança dos alimentos [13, 41, 53-55].

Bas et al. [46] constataram que dentre os 764 manipuladores avaliados, 43,4\% não tinham noções sobre temperaturas adequadas de armazenamento dos alimentos e contaminação cruzada. Marais et al. [56] encontraram um percentual semelhante $(46,0 \%)$ e Osaili et al. ${ }^{[54}$ uma proporção relativamente maior $(69,4 \%)$, valores considerados insatisfatórios pelos autores. Isto sugere que há deficiência no processo de recrutamento, seleção e treinamento dos manipuladores destas empresas [46, 57-59].

De acordo com a RDC no 216, de 15 de setembro de 2004 [60], as operações de higienização devem ser realizadas por funcionários comprovadamente capacitados, de forma a garantir a manutenção e minimizar o risco de contaminação. Os programas de treinamento são mais efetivos para manipuladores de alimentos que trabalham em restaurantes ligados a grandes redes ou franquias, pois, são gerenciados de forma centralizada [61-63]. As redes e franquias geralmente possuem um inspetor ou técnico capacitado e treinado para fiscalizar o cumprimento das 
normas, o que geralmente não acontece com empresas de pequeno e médio porte [61, 62].

Quando se avalia a interação entre treinamentos, conhecimentos, atitude e práticas (CAP) dos manipuladores sobre segurança dos alimentos fatores como ambiente de trabalho, nível de experiência no cargo, carga horária, escolaridade e outras questões sociodemográficas, também devem ser considerados, pois podem condicionar um comportamento negativo em relação a execução de práticas de higiene e resultar em um elevado número de não conformidades [64].

Estudos sobre serviços de alimentação no Brasil mostram o baixo nível de escolaridade dos manipuladores de alimentos [65, 66], situação também verificada em países em desenvolvimento [67, 46] e desenvolvidos [68]. Estes resultados estão em consonância com os resultados obtidos por Medeiros et al. ${ }^{[69]} \mathrm{em}$ relação à escolaridade de manipuladores de alimentos em restaurantes. Fato também observado por Andrade [70] em empresas do setor de turismo. É preocupante a falta de interesse dos gestores sobre a escolaridade, uma vez que o treinamento pode ser oferecido pelo contratante. O nível de escolaridade interfere no aproveitamento do indivíduo nos treinamentos e interpretação de procedimentos, condição indispensável para o desenvolvimento do funcionário $[69,70]$.

Outro fator a ser considerado no treinamento realizado para os manipuladores de alimentos está relacionado carga horária mínima necessária para o tópico de higiene dos alimentos. Pesquisas confirmam a melhoria no conhecimento sobre segurança dos alimentos dos empregados com treinamentos entre 3,7 e 5 horas ${ }^{[69,71]}$ e cerca de 30,5 horas como o tempo ideal médio [72]. A diferença entre as cargas horárias sugeridas pelos autores pode ser justificada pelos fatores que podem intervir na apreensão do conhecimento do público treinado. Além disso, Cunha et al. ${ }^{[65]}$ e McIntyre et al. [68] relatam que os treinamentos devem ser realizados com periodicidade estabelecida (a cada 6 meses ou até 1 ano) pois, o CAP diminue com o passar do tempo, podendo representar riscos aos consumidores. Os atos normativos (federal e estadual) não preveem a periodicidade e a carga horária mínima para formação de manipuladores, apenas estabelecem os tópicos que devem ser abordados [60,73].

A maioria dos estudos indica que os manipuladores de alimentos possuem insuficiente conhecimento das práticas adequadas de higiene dos alimentos [65, 55, 71, 74]. Indicam também a necessidade da elaboração mais criteriosa do planejamento dos treinamentos em relação aos tópicos abordados, o tempo total de execução e os tópicos desenvolvidos, pois, muitos profissionais da área desconhecem a legislação, as ferramentas de qualidade e conceitos importantes (perigo, riscos, microbiologia, entre outros) para evitar a contaminação dos alimentos $[75,67,76,77]$.

O desenvolvimento de políticas de Recursos Humanos (RH), para recrutamento de manipuladores de alimentos, também é de grande relevância para diminuir os riscos de contaminação. Esta política deve estar pautada em todas as esferas da organização (da alta direção ao operador), fornecer recursos para aplicá-las e criar um sistema de documentação e comunicação efetivo [78].

Nieves et al. ${ }^{[79]}$ enfatizam que gerentes do ramo de turismo devem ter a consciência de que o estabelecimento de critérios para contratação de funcionários proporciona a formação de equipes mais capacitadas, com competências e habilidades que influenciam no desenvolvimento de produtos e serviços e na inovação organizacional (técnica, comportamento, desempenho e criatividade). Além disso, equipes mais capacitadas e adaptadas às rotinas específicas do turismo contribuem com a melhoria dos processos e na oferta de serviços mais qualificados aos clientes. As empresas de turismo abertas para a implementação de medidas inovadoras têm obtido significativos resultados na melhoria do desempenho (financeiro, técnico, serviços e outros) e na satisfação de seus clientes ${ }^{[80]}$. Gounaris ${ }^{[81]}$ demonstrou que políticas de RH melhoraram substancialmente o grau de satisfação com o trabalho entre os funcionários do setor hoteleiro. Ruizalba et al. [82] Firth et al. [83] e Silva [84] verificaram associações significativas entre satisfação e comprometimento e concluíram que funcionários com satisfação no trabalho são mais comprometidos com a empresa.

Marco-Lajara e Ubeda-García [32] indicam que uma das principais razões para implementação de estratégias de RH para contratação de funcionários nas empresas hoteleiras está relacionada à alta rotatividade do setor. Os autores explicam que uma das formas de solucionar esse problema seria o estabelecimento de políticas de gestão de recursos humanos definidas e concisas. Ainda, os referidos autores constataram que os maiores percentuais de rotatividade ocorrem entre os funcionários operacionais: manutenção (26,9\%), alimentação $(25,4 \%)$ e recepção $(18,74 \%)$, enquanto os 
menores, correspondem aos gestores $(6,05 \%)$ e pessoal de outras áreas da administração operacional $(5,18 \%)$. Lee et al. [16] e Cho et al. [72] identificaram situações similiares em restaurantes comerciais e hotéis.

Karatepe et al. ${ }^{[85]}$ relatam que as principais razões atribuídas à rotatividade no setor hoteleiro estão envolvidas com ambientes estressantes, elevada carga horária, falta de gerenciamento participativo, trabalho obrigatório em feriados e os baixos salários. Por isso, torna-se imprescindível a gestão de $\mathrm{RH}$ no desenvolvimento das habilidades lógicas e emocionais dos funcionários para enfrentarem estes ambientes adversos e diminuir o nível de insatisfação no trabalho. Em serviços de alimentação, a rotatividade pode ocasionar um declínio da qualidade e segurança do alimento servido gerando perdas de clientes, bem como comprometer a produtividade da empresa por sobrecarga de trabalho para os outros operadores e aumento de despesas relativas a treinamentos e cursos para novos funcionários ${ }^{[70]}$. Além dos problemas de rotatividade, outro fator importante, característico do setor, está relacionado aos salários mais baixos pagos aos funcionários de serviços de alimentação quando comparados aos de outras atividades do mesmo segmento [22]. Esta situação não é exclusiva do Brasil, pois de acordo com estudo de Marco-Lajara e UbedaGarcía [32], na Espanha são observadas características semelhantes em referência às condições de trabalho, rotatividade, recrutamento, capacitação e salários no setor.

No entanto, ainda são poucas as pesquisas sobre a administração desses estabelecimentos e sua relação com a segurança dos alimentos, visando caracterizar os sistemas de gestão da qualidade em restaurantes comerciais, a política de recursos humanos, a competência dos administradores e a qualidade dos treinamentos realizados $[75,9,13,69,86,87]$.

É notório que há muitas falhas, tanto no Brasil como em outros países, relacionadas à execução dos procedimentos de higiene operacional em estabelecimentos de serviços de alimentação de diferentes ramos, conforme descrito anteriormente. No entanto, ainda há pouca percepção dos empresários sobre questões de qualidade e segurança de alimentos. A falta de conhecimento da alta direção sobre as ferramentas da qualidade e seus benefícios, pode acarretar em uma implementação ineficaz, gerando desperdício de tempo e dinheiro e falta de comprometimento de todos os envolvidos ${ }^{[13,35]}$. Assim, o sucesso da aplicação do sistema parece estar mais relacionado ao envolvimento e comprometimento dos participantes do que com o tipo de produto e serviço oferecido ou nível tecnológico alcançado [16, 74, 88, 89].

Para desenvolvimento de um plano adequado de um Sistema de Gestão da Qualidade e Segurança dos Alimentos (SGSA) e sua aplicação em uma unidade hoteleira, primeiramente, é necessário o comprometimento de todos os recursos envolvidos, principalmente da alta direção, pois é de sua responsabilidade estabelecer o planejamento e a política de qualidade a serem implementados. Além disso, é seu papel definir as responsabilidades e autoridades dentro da organização e comunicá-las para assegurar a operação e manutenção do sistema [74, 90]. Embora existam diversas dificuldades na implantação de um Sistema de Gestão da Qualidade em pequenas e médias empresas, o comprometimento da alta direção é que determinará o sucesso ou o fracasso de sua adoção, pois esta disponibiliza os recursos necessários e mobiliza os funcionários para atingir o objetivo determinado [91-93].

Wu [35] destaca que a alta direção é determinante para o sucesso da implementação e criação de estratégias para o desenvolvimento de um SGSA. As ações direcionadas à segurança dos alimentos, apesar de compulsórias, são mais dependentes das iniciativas e da cultura das próprias empresas, isto é, estas verificam a necessidade de desenvolver produtos e serviços de qualidade superior, que lhes proporcionem uma vantagem competitiva no mercado. Entretanto, as empresas esperam que os órgãos públicos elaborem ações que os auxiliem na implementação de um SGSA, proporcionando incentivos para que os custos operacionais sejam diminuídos [13,35]. Verifica-se que quando a implantação de um SGSA é plenamente apoiada pela alta direção são constatadas melhorias estruturais, na formação do pessoal, na rotatividade (absenteísmo) e na capacitação de gerentes. Por isso, é de suma importância avaliar as empresas do setor de turismo, pois, em um mercado competitivo e exigente como o atual, desperdícios de tempo e recursos podem determinar a sobrevivência da empresa no mercado, principalmente, para aquelas que possuem recursos escassos [13, 94].

\section{CONSIDERAÇÕES FINAIS}

Com base no exposto, verifica-se, nos últimos anos, que a demanda pelo consumo de alimentos fora do domicílio, por meio de serviços de alimentação comerciais e vinculados ao turismo, estão em expansão no Brasil. Porém, a capacidade técnica dos 
manipuladores, referindo-se à higiene e à sanidade no preparo das refeições, não vem acompanhando essa tendência, fato demonstrado pelas inúmeras não conformidades encontradas em serviços de alimentação de diversos setores.

Verifica-se que surtos de origem alimentar, geralmente, estão associados aos conhecimentos, atitudes e práticas insatisfatórias dos manipuladores em relação à execução adequada das operações de higiene alimentar, principalmente, sobre aspectos que representam importantes entraves para a segurança do alimento. Esses fatos reforçam a falta de conhecimento e conscientização sobre o assunto e a necessidade de formação contínua dos agentes envolvidos no setor. Além disso, salientam também, a necessidade dos órgãos públicos e autoridades sanitárias realizarem ações educativas e de conscientização como forma de apoio as empresas e aos consumidores, visando o preparo e consumo de alimentos seguros. Somam-se a esses fatores a falta de inspeções regulares dos órgãos fiscalizadores e a capacitação inadequada dos agentes sanitários. Sendo assim, os programas da qualidade, poderiam ser ampliados com a atuação mais efetiva da fiscalização, uma vez que a implantação, no mínimo das Boas Práticas de Higiene é obrigatória para este segmento. Ademais, a implementação de programas de qualidade permite a melhoria do serviço prestado, garante o bem estar dos consumidores, além de proporcionar maior confiabilidade e fidelização dos serviços.

Infere-se, também que os resultados insatisfatórios das condições higiênico-sanitárias constatados em empresas de serviços de alimentação do setor de turismo e outros podem ser justificados pelo inadequado critério de seleção, pela deficiência do programa de capacitação e da avaliação de desempenho dos funcionários para o desenvolvimento pessoal e profissional dos mesmos.

Uma política de recursos humanos direcionada para atender aos anseios dos funcionários poderá motivá-los para o trabalho, permitir o desenvolvimento de serviços de qualidade, e minimizar os problemas enfrentados pelas empresas.

Espera-se que o levantamento bibliográfico realizado constitua subsídios que auxiliem o setor na melhor compreensão do comportamento dos manipuladores nas operações que representem riscos à saúde dos consumidores.

\section{REFERÊNCIAS}

[1] Associação Brasileira das Indústrias de Alimentos (ABIA). 7o Congresso Internacional de Food Service 2014 [internet]. 2014 [acesso em 31 mar 2015]. Disponível em: http://www.abia.org.br/cfs2014/mercado.aspx

[2] Instituto Brasileiro de Geografia e Estatística - IBGE. Pesquisa de Orçamentos Familiares 2008-2009: Despesas, Rendimentos e Condições de Vida. Rio de Janeiro; 2010.

[3] Instituto Brasileiro de Geográfia e Estatística - IBGE. Pesquisa de Orçamentos Familiares 2002-2003: primeiros resultados. Rio de Janeiro; 2004.

[4] Associação Brasileira das Indústrias de Alimentos (ABIA). 6o Congresso Internacional de Food Service 2013 [internet]. 2013 [acesso em 16 jun 2015]. Disponível em: http://www.abia.org.br/cfs2013/

[5] Akutsu R de C, Botelho RA, Camargo EB, Sávio KEO, Araújo WC. Adequação das boas práticas de fabricação em serviços de alimentação. Rev Nutr. 2005;18(3):419-427.

[6] Brasil. Ministério do Turismo. Brasil comemora avanços no Dia Mundial do Turismo [internet]. Brasilia; 2013 [acesso em 31 mar 2015]. Disponível em: http://www.turismo.gov.br/turismo/noticias/todas_noticia s/20130925-1.html

[7] Portal da Copa. Brasil terá pelo menos 422 novos empreendimentos de hospedagem até 2016 [internet]. 2014 [acesso em 31 mar 2015]. Disponível em: http://www.copa2014.gov.br

[8] Serviço de Apoio às Micro e Pequenas Empresas SEBRAE. Anuário do trabalho na micro e pequena empresa: 2013. 6ạ ed. São Paulo; 2013.

[9] Cavalli SB, Salay E. Gestão de pessoas em unidades produtoras de refeições comerciais e a segurança alimentar. Rev Nutr. 2007;20(6):657-667.

[10] Panchal PK, Bonhote P, Dworkin MS. Food Safety Knowledge among Restaurant Food Handlers in Neuchâtel, Switzerland. Food Prot Trends. 2013;33(3):133-144.

[11] Pichler J, Ziegler J, Aldrian U, Allerberger F. Evaluating levels of knowledge on food safety among food handlers from restaurants and various catering businesses in Vienna, Austria 2011/2012. Food Control. 2014;35(1):34 40.

[12] Martins RB, Ferreira D, Moreira LM, Hogg T, Gestal J. Knowledge on food hygiene of food service staff working in nursing homes and kindergartens in Porto region - Portugal. Food Control. 2014;42:54-62. 
[13] Ko WH. The relationship among food safety knowledge, attitudes and self-reported HACCP practices in restaurant employees. Food Control. 2013;29(1):192-197.

[14] Luber P. Cross-contamination versus undercooking of poultry meat or eggs - which risks need to be managed first? Int J Food Microbiol. 2009;134(1-2):21-8.

[15] Medeiros CO, Cavalli SB, Salay E, Proença RPC. Assessment of the methodological strategies adopted by food safety training programmes for food service workers: A systematic review. Food Control. 2011;22(8):1136-1144.

[16] Lee JE, Almanza BA, Jang S, Nelson DC, Ghiselli RF. Does transformational leadership style influence employees' attitudes toward food safety practices? Int J Hosp Manag. 2013;33(1):282-293.

[17] Liu Z, Zhang G, Zhang X. Urban street foods in Shijiazhuang city, China: Current status, safety practices and risk mitigating strategies. Food Control. 2014;41(1):212-218.

[18] Brasil. Ministério da Saúde. Vigiância Epidemiológica das Doenças Transmitidas por Alimentos - VE-DTA [internet]. 2014 [acesso em 31 mar 2015]. Disponível em: http://www.anrbrasil.org.br/new/pdfs/2014/3_PAINEL_ 1_ApresentacaoRejaneAlvesVigilanciaEpidemiologica-VEDTA-Agosto_2014_PDF.pdf

[19] Galeazzi IMS, Garcia L, Driemeier MM, Toni M De, Kreling NH, Follador P. Mulheres trabalhadoras: 10 anos de mudanças no mercado de trabalho atenuam desigualdades. Mulh e Trab. 2003;3:10-35.

[20] Instituto Brasileiro de Geográfia e Estatística - IBGE. Mulher no Mercado de Trabalho: perguntas e respostas. Rio de Janeiro; 2012.

[21] Savio KEO. Perfil nutricional da clientela atendida em restaurantes vinculados ao Programa de Alimentação do Trabalhador do Distrito Federal, Brasil [dissertação]. Brasília: Universidade de Brasilia; 2002.

[22] Instituto Brasileiro de Geográfia e Estatística - IBGE. Economia do turismo: uma perspectiva macroeconômica 2003-2009. Rio de Janeiro; 2012.

[23] Associação Brasileira de Bares e Restaurantes (ABRASEL). Alimentação concentra mais empresas e lidera em faturamento em serviços [internet]. 2012 [acesso em 16 jun 2015]. Disponível em: http://www.abrasel.com.br

[24] Food and Agriculture Organization of the United Nations (FAO). FAO/WHO guidance to governments on the application of HACCP in small and/or less-developed food businesses; 2006.
[25] Instituto Brasileiro de Geografia e Estatística (IBGE). Micro e pequenas empresas geram 27\% do PIB do Brasil [internet]. 2014 [acesso em 16 jun 2015]. Disponível em: http://www.sebraesp.com.br

[26] Instituto Brasileiro de Geografia e Estatística (IBGE). Empresas de serviços serão a maioria no mercado paulista em 2020 [internet]. 2012 [acesso em 16 jun 2015]. Disponível em: http://www.sebraesp.com.br

[27] Associação Brasileira das Indústrias de Alimentos (ABIA). Balanço anual 2005 e perspectivas para 2006 [internet]. 2006 [acesso em 16 jun 2015]. Disponível em: http://www.abia.org.br/anexos/BalancoAnual2005.pdf

[28] Associação Brasileira das Indústrias de Alimentos (ABIA). I Congresso Internacional de Food Service 2008 [internet]. 2008 [acesso em 16 jun 2015]. Disponível em: http://www.abia.org.br/congressofoodservice2008/telas/fo od-service.asp

[29] Associação Brasileira das Indústrias de Alimentos (ABIA). Relatório Anual 2008 [internet]. 2008 [acesso em 16 jun 2015]. Disponível em: http://www.ncbi.nlm.nih.gov/pubmed/19241998

[30] Associação Brasileira das Indústrias de Alimentos (ABIA). O setor em números [internet]. 2013 [acesso em 16 jun 2015]. Disponível em: http://www.abia.org.br/vs/setoremnumeros.aspx

[31] Cônsoli MA. Análise dos Aspectos Relevantes para Integração na Cadeia de Suprimentos Aplicada ao Setor de Serviços de Alimentação [tese]. São Carlos: Escola de Engenharia, Universidade de São Paulo; 2009.

[32] Marco-Lajara B, Úbeda-García M. Human resource management approaches in Spanish hotels: An introductory analysis. Int J Hosp Manag. 2013;35:339-347.

[33] Portal da Copa. Economia do turismo cresce no Brasil [internet]. 2014 [acesso em 16 jun 2015]. Disponível em: http://www.copa2014.gov.br/pt-br/noticia/economia-doturismo-cresce-no-brasil

[34] Brasil. Ministério do Turismo. Estatísticas Básicas de Turismo [internet]. 2014 [acesso em 16 jun 2015]. Disponível em: http://www.dadosefatos.turismo.gov.br

[35] Wu SL. Factors influencing the implementation of food safety control systems in Taiwanese international tourist hotels. Food Control. 2012;28(2):265-272.

[36] Food and Agriculture Organization of the United Nations (FAO). Estrategia de la FAO relativa al enfoque de calidad e inocuidad de los alimentos basado en la cadena 
alimentaria: documento marco para la formulación de la futura orientación estratégica [internet]. 2003 [acesso em 16 jun 2015]. Disponível em: http://www.fao.org/docrep/meeting/006/y8350s.htm

[37] Williams CJ, Schenkel K, Eckmanns T, Altmann D, Krause G. FIFA World Cup 2006 in Germany: enhanced surveillance improved timeliness and detection. Epidemiol Infect. 2009;137(4):597-605.

[38] Willis C, Elviss N, Aird H, Fenelon D, McLauchlin J. Evaluation of hygiene practices in catering premises at largescale events in the UK: Identifying risks for the Olympics 2012. Public Health. 2012;126(8):646-656.

[39] Jones TF, Grimm K. Public Knowledge and Attitudes Regarding Public Health Inspections of Restaurants. Am J Prev Med. 2008;34(6):510-513.

[40] Silva Junior EA. Manual de controle higiênico-sanitário em serviços de alimentação. 7a ed. São Paulo: Varela; 2014.

[41] Luning PA, Chinchilla AC, Jacxsens L, Kirezieva K, Rovira J. Performance of safety management systems in Spanish food service establishments in view of their context characteristics. Food Control. 2013;30(1):331-340.

[42] Baş M, Yüksel M, Çavuşoğlu T. Difficulties and barriers for the implementing of HACCP and food safety systems in food businesses in Turkey. Food Control. 2007;18(2):124 130.

[43] Fielding L, Ellis L, Beveridge C, Peters A. An evaluation of HACCP implementation status in UK small and medium enterprises in food manufacturing. Int J Environ Health Res. 2005;15(2):117-126.

[44] Luning PA, Marcelis WJ. A conceptual model of food quality management functions based on a techno-managerial approach. Trends Food Sci Technol. 2007;18(3):159-166.

[45] Food and Drug Administration. Report of the FDA retail food program program database of foodborne illness risk factors; 2000.

[46] Baş M, Şafak Ersun A, Kıvanç G. The evaluation of food hygiene knowledge, attitudes, and practices of food handlers' in food businesses in Turkey. Food Control. 2006;17(4):317322.

[47] Cardoso RCV, Góes JÂW, Almeida RCC, Guimarães AG, Barreto DL, da Silva SA, et al. Programa nacional de alimentação escolar: há segurança na produção de alimentos em escolas de Salvador (Bahia)? Rev Nutr. 2010;23(5):801811.
[48] Campos AKC, Cardonha ÂMS, Pinheiro LBG, Ferreira NR, Azevedo PRM, Stamford TLM. Assessment of personal hygiene and practices of food handlers in municipal public schools of Natal, Brazil. Food Control. 2009;20(9):807-810.

[49] Costa RA, Mourão JA, Ângelo ÂMO, Vieira GHF, Alves LAO. Análise bacteriológica de merenda escolar servida em um colégio estadual de Sobral, Ceará. Hig Aliment. 2008;22(166/167):138-141.

[50] Danelon MS, Silva MV. Análise das condições higiênicosanitárias das áreas de preparo e consumo de alimentos, disponíveis para alunos de escolas públicas e privadas. Hig Aliment. 2007;21(152):25-30.

[51] Farche LM, Pereira CHC, Castro GPP, Pelizer LH. O panorama higiênico-sanitário nas cozinhas das escolas da rede pública de Franca, SP. Hig Aliment. 2007;21(154):27-29.

[52] Lockis VR, Cruz AG, Walter EHM, Faria JAF, Granato D, Sant'Ana AS. Prerequisite programs at schools: diagnosis and economic evaluation. Foodborne Pathog Dis. 2011;8(2):213-220.

[53] Lazou T, Georgiadis M, Pentieva K, McKevitt A, Iossifidou E. Food safety knowledge and food-handling practices of Greek university students: A questionnaire-based survey. Food Control. 2012;28(2):400-411.

[54] Osaili TM, Abu Jamous DO, Obeidat BA, Bawadi HA, Tayyem RF, Subih HS. Food safety knowledge among food workers in restaurants in Jordan. Food Control. 2013;31(1):145-150.

[55] Cunha DT, Stedefeldt E, De Rosso VV. Perceived risk of foodborne disease by school food handlers and principals: The influence of frequent training. J Food Saf. 2012;32(2):219-225.

[56] Marais M, Conradie N, Labadarios D. Small and micro enterprises - aspects of knowledge, attitudes and practices of managers' and food handlers' knowledge of food safety in the proximity of Tygerberg Academic Hospital, Western Cape. South African J Clin Nutr. 2008;20(2):50-61.

[57] Martins RB, Hogg T, Otero JG. Food handlers' knowledge on food hygiene: The case of a catering company in Portugal. Food Control. 2012;23(1):184-190.

[58] Seaman P. Food hygiene training: Introducing the Food Hygiene Training Model. Food Control. 2010;21(4):381-387.

[59] Powell DA, Erdozain S, Dodd C, Costa R, Morley K, Chapman BJ. Audits and inspections are never enough: A critique to enhance food safety. Food Control. 2013;30(2):686-691. 
[60] Brasil. Ministério da Saúde. Agência Nacional de Vigilância Sanitária. Resolução RDC no 216, de 15 de setembro de 2004. Dispõe sobre Regulamento Técnico de Boas Práticas para Serviços de Alimentação. Diário Oficial da União. 16 set 2004.

[61] Harris KJ, DiPietro RB, Murphy KS, Rivera G. Critical food safety violations in Florida: Relationship to location and chain vs. non-chain restaurants. Int J Hosp Manag. 2014;38:57-64.

[62] Kassa H, Silverman GS, Baroudi K. Effect of a manager training and certification program on food safety and hygiene in food service operations. Environ Health Insights. 2010;4:13-20.

[63] Murphy KS, DiPietro RB, Kock G, Lee JS. Does mandatory food safety training and certification for restaurant employees improve inspection outcomes? Int J Hosp Manag. 2011;30(1):150-156.

[64] Kibret M, Abera B. The sanitary conditions of food service establishments and food safety knowledge and practices of food handlers in bahir dar town. Ethiop J Health Sci. 2012;22(1):27-35.

[65] Cunha DT, Stedefeldt E, de Rosso VV. The role of theoretical food safety training on Brazilian food handlers' knowledge, attitude and practice. Food Control. 2014;43:167-174.

[66] Nunes BN, Cruz AG, Faria JAF, Sant'Ana AS, Silva R, Moura MRL. A survey on the sanitary condition of commercial foods of plant origin sold in Brazil. Food Control. 2010;21(1):50-54.

[67] Abdul-Mutalib NA, Abdul-Rashid MF, Mustafa S, Amin-Nordin S, Hamat RA, Osman M. Knowledge, attitude and practices regarding food hygiene and sanitation of food handlers in Kuala Pilah, Malaysia. Food Control. 2012;27(2):289-293.

[68] McIntyre L, Peng D, Henderson SB. Retraining effectiveness in FOODSAFE trained food handlers in British Columbia, Canada. Food Control. 2014;35(1):137-141.

[69] Medeiros CO, Cavalli SB, da Costa Proença RP. Human resources administration processes in commercial restaurants and food safety: The actions of administrators. Int J Hosp Manag. 2012;31(3):667-674.

[70] Andrade ML. Segurança dos alimentos no setor hoteleiro: caracterização das empresas e avaliação da percepção dos agentes envolvidos - estudo de caso [dissertação]. Piracicaba: Universidade de São Paulo; 2014.
[71] McIntyre L, Vallaster L, Wilcott L, Henderson SB, Kosatsky T. Evaluation of food safety knowledge, attitudes and self-reported hand washing practices in FOODSAFE trained and untrained food handlers in British Columbia, Canada. Food Control. 2013;30(1):150-156.

[72] Cho S, Woods RH, (Shawn) Jang S, Erdem M. Measuring the impact of human resource management practices on hospitality firms' performances. Int J Hosp Manag. 2006;25(2):262-277.

[73] São Paulo. Secretaria de Estado da Saúde. Portaria CVS no 5 , de 9 de abril de 2013. Aprova o regulamento técnico sobre boas práticas para estabelecimentos comerciais de alimentos e para serviços de alimentação, e o roteiro de inspeção, anexo. Diário Oficial do Estado de São Paulo. 19 abr 2013.

[74] Abdullah Sani N, Siow ON. Knowledge, attitudes and practices of food handlers on food safety in food service operations at the Universiti Kebangsaan Malaysia. Food Control. 2014;37:210-217.

[75] Bolton DJ, Meally A, Blair IS, McDowell DA, Cowan C. Food safety knowledge of head chefs and catering managers in Ireland. Food Control. 2008;19(3):291-300.

[76] Jianu C, Chiş C. Study on the hygiene knowledge of food handlers working in small and medium-sized companies in western Romania. Food Control. 2012;26(1):151-156.

[77] Tokuç B, Ekuklu G, Berberoğlu U, Bilge E, Dedeler H. Knowledge, attitudes and self-reported practices of food service staff regarding food hygiene in Edirne, Turkey. Food Control. 2009;20(6):565-568.

[78] Szabo EA, Porter WR, Sahlin CL. Outcome based regulations and innovative food processes: An Australian perspective. Innov Food Sci Emerg Technol. 2008;9(2):249_ 254.

[79] Nieves J, Quintana A, Osorio J. Knowledge-based resources and innovation in the hotel industry. Int J Hosp Manag. 2014;38:65-73.

[80] Grissemann U, Plank A, Brunner-Sperdin A. Enhancing business performance of hotels: The role of innovation and customer orientation. Int J Hosp Manag. 2013;33(1):347-356.

[81] Gounaris S. The notion of internal market orientation and employee job satisfaction: some preliminary evidence. J Serv Mark. 2008;22(1):68-90.

[82] Ruizalba JL, Bermúdez-González G, Rodríguez-Molina MA, Blanca MJ. Internal market orientation: An empirical research in hotel sector. Int J Hosp Manag. 2014;38:11-19. 
[83] Firth L, Mellor DJ, Moore KA, Loquet C. How can managers reduce employee intention to quit? J Manag Psychol. 2004;19(2):170-187.

[84] Silva P. Effects of disposition on hospitality employee job satisfaction and commitment. Int J Contemp Hosp Manag. 2006;18(4):317-328.

[85] Karatepe OM, Yorganci I, Haktanir M. Outcomes of customer verbal aggression among hotel employees. Int J Contemp Hosp Manag. 2009;21(6):713-733.

[86] Hedberg CW, Smith SJ, Kirkland E, Radke V, Jones TF, Selman CA. Systematic environmental evaluations to identify food safety differences between outbreak and nonoutbreak restaurants. J Food Prot. 2006;69(11):2697-2702.

[87] Van Tonder I, Lues JFR, Theron MM. The personal and general hygiene practices of food handlers in the delicatessen sections of retail outlets in South Africa. J Environ Health. 2007;70(4):33-38.

[88] Garayoa R, Díez-Leturia M, Bes-Rastrollo M, GarcíaJalón I, Vitas AI. Catering services and HACCP: Temperature assessment and surface hygiene control before and after audits and a specific training session. Food Control. 2014;43:193-198.
[89] Spexoto AA. Aplicação do sistema de análise de perigos e pontos críticos de controle (APPCC) em propriedades leiteiras [dissertação]. Pirassununga: Universidade de São Paulo; 2003.

[90] Nieto-Montenegro S, Brown JL, LaBorde LF. Development and assessment of pilot food safety educational materials and training strategies for Hispanic workers in the mushroom industry using the Health Action Model. Food Control. 2008;19(6):616-633.

[91] Figueiredo VF. Modelo de sistema de gestão da segurança de alimentos na pequena e média empresa [tese]. São Paulo: Universidade de São Paulo; 2005.

[92] Jevšnik M, Hlebec V, Raspor P. Food safety knowledge and practices among food handlers in Slovenia. Food Control. 2008;19(12):1107-1118.

[93] Taylor E. HACCP in small companies: benefit or burden? Food Control. 2001;12(4):217-222.

[94] Rodgers S. Applied research and educational needs in food service management. Int J Contemp Hosp Manag. 2005;17(4):302-314. 